\title{
An Immunosuppression Hidden Malignancy: Case Report of An Unexpected Clostridium Septicum Infection and Its Fatal Consequence
}

ISSN: 2637-7632

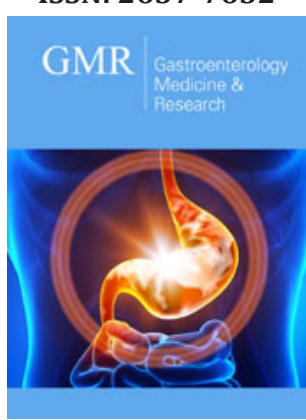

*Corresponding author: Grade $\mathrm{M}$, Department of Gastroenterology, Infectious Diseases and General Internal Medicine Christian Hospital Quakenbrück, Germany

Submission: 価 May 14, 2021

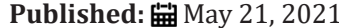

Volume 5 - Issue 5

How to cite this article: Grade M, Welk E, Sostmann H, Hammerstein HV, McAulay CH, Bostelmann R. An Immunosuppression Hidden Malignancy: Case Report of An Unexpected Clostridium Septicum Infection and Its Fatal Consequence. Gastro Med Res. 5(5). GMR. 000625. 2021.

DOI: 10.31031/GMR.2021.05.000625

Copyright@ Grade M, This article is distributed under the terms of the Creative Commons Attribution 4.0 International License, which permits unrestricted use and redistribution provided that the original author and source are credited.
Grade $\mathbf{M}^{1 *}$, Welk $\mathbf{E}^{1}$, Sostmann $\mathbf{H}^{1}$, Hammerstein $\mathrm{HV}^{1}$, McAulay $\mathrm{CH}^{3}$ and Bostelmann $\mathrm{R}^{2}$

${ }^{1}$ Department of Gastroenterology, Infectious Diseases and General Internal Medicine Christian Hospital Quakenbrück, Germany

${ }^{2}$ Department of Neurosurgery and Spine Surgery, Christian Hospital Quakenbrück, Germany ${ }^{3}$ Department of Radiology, St. Marienhospital, Vechta, Germany

\begin{abstract}
Background: Clostridium septicum is a gram-positive, spore-forming bacteria belonging to the human intestinal flora. Infections are rare but lead to high mortality and are associated with immunosuppression, malignancy and multipathogenic infections. We present the case of an immunocompromised patient in which an inapparent Clostridium septicum infection and colorectal cancer are simultaneously detected.

Case presentation: A 67-year-old man was admitted to our hospital with fatigue and inappetence. Past medical history included lupus erythematosus, treated with prednisolone, azathioprine, and hydroxychloroquine, and coronary heart disease managed through stenting. His CRP was raised at $321 \mathrm{mg} / \mathrm{l}$ (norm.: $<5 \mathrm{mg} / \mathrm{l}$ ) and procalcitonin elevated at $0.65 \mathrm{ng} / \mathrm{ml}$ (norm.: $<0.005 \mathrm{ng} / \mathrm{ml}$ ) with a haemoglobin concentration of 9.5g/dl (norm.: $14-18 \mathrm{mg} / \mathrm{dl}$ ). Clostridium septicum and Staphylococcus saccharolyticus were both isolated from anaerobic blood cultures and the decision was taken to commence antibiotic treatment with clindamycin and piperacillin/tazobactam. Inflammatory markers decreased as a result. Additional results obtained from colonoscopy revealed adenocarcinoma of the ascending colon. The patient later went on to receive a right-sided hemicolectomy during elective surgery after developing impaired hemodynamic response due to gastrointestinal bleeding. Following a complete course of antibiotic therapy, the patient recovered from his surgery and the infection.

Conclusion: The detection of infection with Clostridium septicum has clinical relevance due to its association with colon cancer. Once the bacteria have been identified, colonoscopy and antibiotic therapy are of vital importance. Immunocompromised patients may have a worsened prognosis, as they often do not show clinical signs of infection, and as a result the diagnosis of a Clostridium septicum and associated malignancy can be delayed or even missed.
\end{abstract}

Keywords: Clostridium septicum; Association colon carcinoma; Bacteremia; Immunosuppression; Inapparent Clostridium septicum infection

Abbreviation: CS: Clostridium Septicum

\section{Introduction}

Clostridium septicum (CS) belongs to the gas-producing bacterium and occurs naturally in the soil as well as the intestinal flora. They are toxin-producing, gram-positive, obligatory anaerobic and form spores under certain conditions. Only $1 \%$ of all blood culture samples are positive for Clostridia and among them CS makes up to $1.3 \%$ of the total [1]. Proven CS in blood cultures is associated with gastrointestinal and hematological malignancy, immunosuppression and diabetes mellitus [2-4]. Related infection mortalities appear to be over $50 \%[4,5]$. Cases are reported highlighting the correlation between immunosuppression and CS as well as CS and colon cancer. We present a rare clinical case in which an unexpected colon cancer was detected alongside a CS infection in an immunocompromised patient.

\section{Case Presentation}

A 67-year-old man was referred to our department by his general practitioner for clarifying the cause of anaemia, fatigue and inappetence. The patient was in a moderate condition and complained of an unexpected weight loss of $7 \mathrm{~kg}$ in 3 months. He revealed a 
40 pack-year smoking history and admitted to eating meat daily. Pre-existing illnesses were lupus erythematosus (treated with $10 \mathrm{mg}$ prednisolone, 400mg hydroxychloroquine and $100 \mathrm{mg}$ azathioprine), arterial hypertension and coronary artery disease treated with atorvastatin, aspirin and clopidogrel. His physical examination was normal, but the laboratory results showed a reduced haemoglobin concentration of 9.5g/dl (norm.: 14-18mg/ $\mathrm{dl}$ ), elevated CRP of $140 \mathrm{mg} / \mathrm{l}$ (norm.: $<5 \mathrm{mg} / \mathrm{l}$ ), raised procalcitonin of $0.26 \mathrm{ng} / \mathrm{ml}$ (norm.: $<0.005 \mathrm{ng} / \mathrm{ml}$ ) and a high ESR over $120 \mathrm{~mm} /$ hr (norm.: 15mm/hr). Blood cultures were sent immediately. Sonography and x-ray examination did not reveal any relevant findings. The decision was taken to commence antibiotic therapy with piperacillin / tazobactam after further elevations of CRP $(321 \mathrm{mg} / \mathrm{l})$ and procalcitonin $(0.65 \mathrm{ng} / \mathrm{ml})$. The following day, blood cultures showed evidence of Staphylococcus saccharloyticus and Clostridium septicum. We added clindamycin whilst infection parameters decreased and proceeded with gastrointestinal endoscopy. A biopsy was taken which revealed adenocarcinoma stage T4a (infiltration of the serosa) N0 M0 G2 V0 L0 and UICC stadium IVa (Figure 1). The decision to commence surgery was made after treating the infection. On the same night the patient lost blood, developed peritonitis and became hemodynamically unstable with a blood pressure of $60 / 40 \mathrm{mmHg}$. An emergency laparoscopy and right-sided hemicolectomy was performed. Intraoperatively, the tumor demonstrated infiltration of the mesenteric root with formation of an abscess cavity. A bacterial smear was taken and lavage and hemicolectomy with double ileostomy were performed. E. coli and Bac. fragilis were found on the smear and identified as being sensible to piperacillin/tazobactam. The patient made a full surgical recovery after receiving a continued antibiotic course. Additional outpatient chemotherapy was recommended.

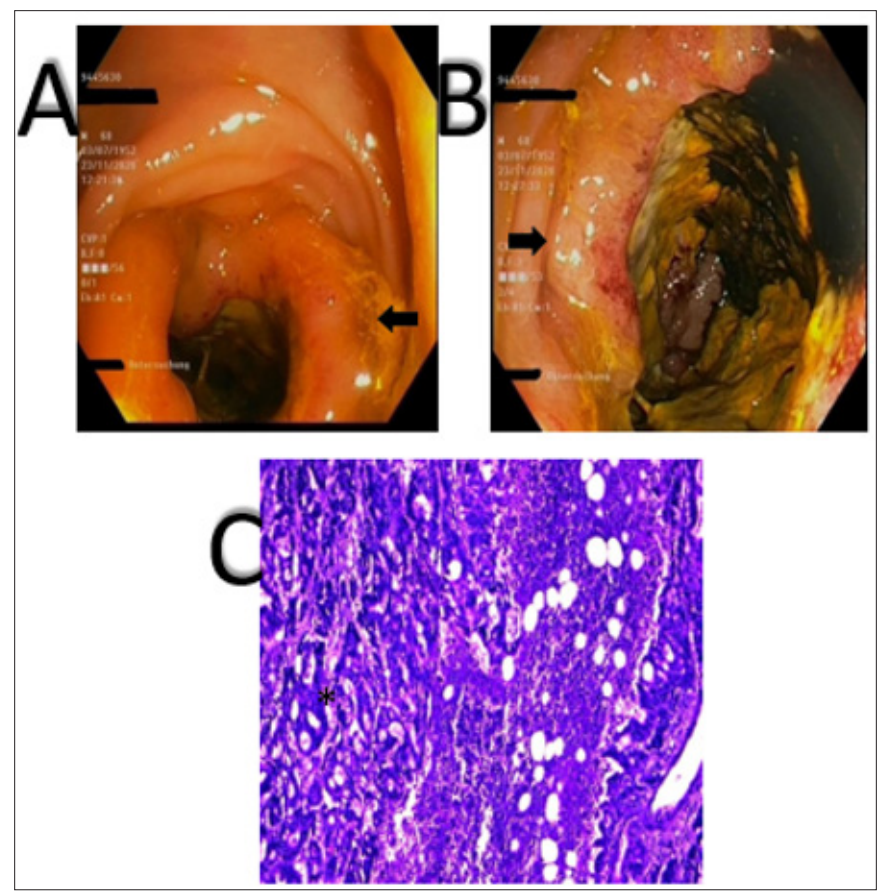

Figure 1: Results of the coloscopy (A, B) with circular growing tumor and histology (C) with atypical glandular cell infiltration from the right colon.

\section{Discussion}

The clinical manifestation of Clostridium septicum infections can vary. The patient in our hospital showed anaemia, inappetence and fatigue. Further cases have described associations with aortic aneurysm 6, the formation of gas gangrene [7] and even pneumocephalus [8]. As a result, diagnosis, treatment and complications may vary and the detection of CS can be forgotten. Early antibiotic treatment has been shown to improve outcomes. The most effective antibiotics are clindamycin, penicillin, metronidazole and moxalactam [1]. We chose clindamycin and the infection parameters decreased. However, the patient became haemodynamic unstable, after blood loss and the onset of peritonitis, before we could completely cure the bacteremia. E. coli and Bac. fragilis were found intraoperatively. Some cases have shown a correlation between CS, perforation and multipathogenic infection with bacteria including Streptococcus bovis, Fusobacterium spp. as well as E. coli and Bac. fragilis [9-11]. The patient we present has a history of systemic lupus erythematosus and takes prednisolone, hydroxychloroquine and azathioprine. The drugs intervene with the immune system in different ways in order to achieve effective immunosuppression [12]. Cellular and humoral immune response are reduced with prednisolone by inhibiting intracellular NF-pathways [13]. Hydroxychloroquine affects toll like receptor-mediated activation of the innate immune response [14] and azathioprine belongs to the group of purine analogues which reduce DNS replication and proliferation of CD3+T cells. Therefore TNF- $\alpha$ release is decreased [15]. A correlation between immunosuppression and bacteraemia with CS has been described $[3,4]$. That may have increased the risk 
of asymptomatic CS appearance in this case. The detection of CS has great clinical significance. It demonstrates a symptomatic course, is associated with gastrointestinal and hematological malignancy and has been shown to have high mortality rates. An early diagnosis and treatment of the bacteremia could improve patient outcomes. Infection in immunocompromised patients may however be clinical inapparent. Therefore, the diagnosis of this bacteria and associated malignancy and complications can be difficult or even fail.

\section{Learning points}

1. Clostridum septicum is strongly associated with malignancy. Colonoscopy is therefore indicated

2. Immunusuppression may hide infection due to Clostridium septicum and therefore the associated malignancy. Missing the microbiological diagnosis may lead to the misinterpretation of malignant tumors.

\section{Conflict of interest}

The authors declare that they have no conflicts of interests.

M. Grade and E. Welk contributed equally to this article.

\section{References}

1. Bodey GP, Rodriguez S, Fainstein V, Elting LS (1991) Clostridial bacteremia in cancer patients. A 12- year experience. Cancer 67(7): 1928-1942.

2. Alpern RJ, Dowell VR (1969) Clostridium septicum infections and malignancy. JAMA 209(3): 385-388.

3. Mirza NN, McCloud JM, Cheetham MJ (2009) Clostridium septicum sepsis and colorectal cancer-a reminder. World J Surg Oncol 7:73.
4. Larson CM, Bubrick MP, Jacobs DM, West MA (1995) Malignancy, mortality, and medicosurgical management of Clostridium septicum infection. Surgery 118(4): 592-598.

5. Mao E, Clements A, Feller E (2011) Clostridium septicum sepsis and colon carcinoma: Report of 4 cases. Case Rep Med 2011:248453.

6. Lehman B, Miller RM, Richter SS, Keller G, Tan C, et al. (2020) Clostridium septicum-infected aortic aneurysm or graft is a deadly diagnosis. J Vasc Surg 71(5): 1781-1788.

7. Srivastava I, Aldape MJ, Bryant AE, Stevens DL (2017) Spontaneous $C$. septicum gas gangrene: A literature review. Anaerobe 48: 165-171.

8. Katyal A, Dmello D (2016) Clostridium septicum pneumocephalus. Neurocrit Care 24(2): 264-267.

9. Gagnière J, Raisch J, Veziant J, Barnich N, Bonnet R, et al. (2016) Gut microbiota imbalance and colorectal cancer. World J Gastroenterol 22(2): 501-518.

10. Kwong TNY, Wang X, Nakatsu G, Chow TC, Tipoe T, et al. (2018) Association between bacteremia from specific microbes and subsequent diagnosis of colorectal cancer. Gastroenterology 155(2): 383-390.e8.

11. Powell MJ, Sasapu KK, Macklin C (2008) Metastatic gas gangrene and colonic perforation: A case report. World J Emerg Surg 3: 15.

12. Kuhn A, Bonsmann G, Anders H-J, Herzer P, Tenbrock K, et al. (2015) The diagnosis and treatment of systemic lupus erythematosus. Dtsch Arztebl Int 112(25): 423-432.

13. Sun F, Zhang Y, Li Q (2017) Therapeutic mechanisms of ibuprofen, prednisone and betamethasone in osteoarthritis. Mol Med Rep 15(2): 981-987.

14. Wallace DJ, Gudsoorkar VS, Weisman MH, Venuturupalli SR (2012) New insights into mechanisms of therapeutic effects of antimalarial agents in SLE. Nat Rev Rheumatol 8(9): 522-533.

15. Giuffrida P, Di Sabatino A (2020) Targeting T cells in inflammatory bowel disease. Pharmacol Res 159: 105040. 